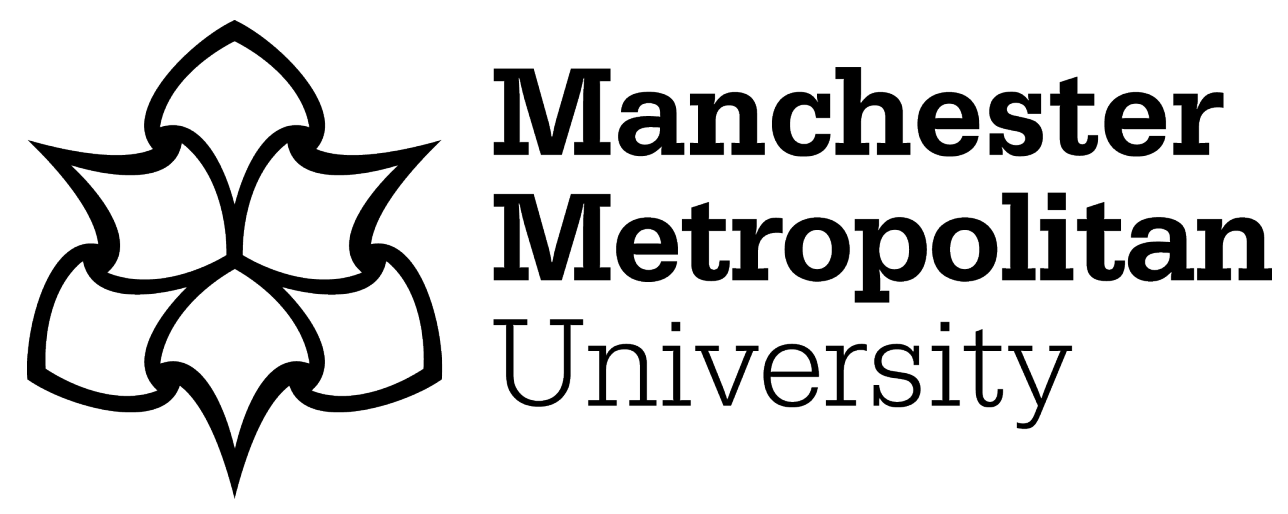

Sharaby, Y, Lynch, S, Joshi, A and Hassan, SS (2014) Bistable dynamics beyond rotating wave approximation. Journal of Nonlinear Optical Physics and Materials, 23 (2). p. 1450019. ISSN 0218-8635

Downloaded from: https://e-space.mmu.ac.uk/842/

Version: Accepted Version

Publisher: World Scientific Publishing

DOI: https://doi.org/10.1142/S0218863514500192

Please cite the published version 


\section{Bistable Dynamics Beyond Rotating Wave Approximation}

$$
\text { Y.A.Sharaby }{ }^{1, a^{*}}, \text { S. Lynch }{ }^{2}, \text { A. Joshi }{ }^{3} \text { and S.S.Hassan }{ }^{2,4}
$$

${ }^{1}$ Suez University, Faculty of Applied Sciences, Physics Department, Suez, Egypt (Yasser_Sharaby@hotmail.com).

2 School of Computing, Mathematics \& Digital Technology, Manchester Metropolitan University, John Dalton Building, Chester St., Manchester M1 5GD, UK (s.lynch@mmu.ac.uk).

${ }^{3}$ Department of Physics, Eastern Illinois University, Charleston, IL61920, USA (ajoshi@eiu.edu).

${ }^{4}$ University of Bahrain, College of Science, Department of Mathematics, P.O. Box 32038 Bahrain (Shoukryhassan@hotmail.com).

${ }^{*}$ Corresponding author.

a Salman bin Abdulaziz University, College of Arts and Sciences, Mathematics Department, Wadi Addwasir, Saudi Arabia. 


\begin{abstract}
We investigate the nonlinear dynamical behavior of optical bistability (OB) for homogeneously broadened two-level atomic media interacting with a single ring cavity mode without invoking the rotating wave approximation (RWA) in the dispersive regime. The periodic oscillations (self-pulsing) and chaos of the unstable state of the $\mathrm{OB}$ curve is affected by the non-vanishing counter rotating terms through the appearance of spikes in its periods. Further, bifurcation with atomic detuning, within and outside the RWA, shows that the OB system can be converted from chaos to self pulsing and vice-versa. Gaussian transverse field structure leads to....
\end{abstract}

\title{
Keywords
}

Optical bistability - bifurcation - rotating wave approximation - self pulsing chaos. 


\section{Introduction}

Optical bistability (OB) phenomena has a vital role in nonlinear optics, both experimentally and theoretically [1-13], because of its potential applications that yield high speed all optical signal processing and all optical computing. Optically bistable elements, those based upon the generation of two stable field states for the same input field, can be used as logic gates, memory devices, switches and differential amplifiers, components in optical computers and communication systems [5, 14-15]. Specifically, the many promising technological applications of the bistable phenomenon in all optical telecommunication networks and optical computing in nonlinear semiconductor lasers and laser amplifiers have been recently reviewed [16]. Further, recent topics such as quantum computation, quantum information processing and entanglement would at a certain level, benefit from further study of $\mathrm{OB}$ systems within the context of cooperative atomic behavior [17]. Optical chaos generated in nonlinear optical systems is applicable for communication information at high data rates [18] and is used in applications of random number generation [19].

Earlier authors [7, 20-21] have applied a linear stability analysis for absorptive optical bistability in a ring cavity and showed that, under certain conditions, part of the upper branch is unstable. In this case, the system can operate as a transformer of continuous wave light into pulsed, where a self pulsing oscillation occurs with a period equal to the cavity round-trip time. This analysis was generalized in the dispersive case [22] (see [11] and references therein). Another mechanism of instability was theoretically studied by Ikeda et.al. [23] where periodic instabilities and chaotic behavior may occur in optical bistable two-level 
systems in a ring cavity with time delay effects, when the time of radiation to propagate from the output- to the input-end is longer than the atomic decay time. This instability can lead to period doubling and chaos as that observed in [3] for Fabry-Perot cavity containing sodium atoms in the absorptive case. Single mode instabilities ranging from gain based laser systems [24] to passive two-level optical bistable systems have been investigated [25] and revisited with more details [26]. Effects of the squeezed vacuum on the dynamic behavior of twolevel OB systems have been discussed in [27]. Recently, novel instability of OB systems of very thin material (of thickness much smaller than the wavelength) [28] has been discussed in a purely absorptive OB Fabry-Perot cavity with multilongitudinal mode depending on the relative position of the thin absorber in the cavity.

Self-pulsing and chaos has been exhibited for three-level atoms interacting with multiple fields [29, 30], probe field experiencing feedback [31], with single mode field in ring cavity environment with squeezed vacuum field [32] and two-photon atomic transitions [33,34]. Recently, the authors of [35] investigated a novel type of instability different from earlier instabilities of $\mathrm{OB}[23,36-38]$ in which the self-pulsing occurrence does not require finite detuning of either the bistable field coupling the atom or the cavity detuning [39], and occurs without involving coupling to multiple modes of the cavity field. Further, instability examination in the lower cooperative branch for three $\wedge$ - and $\mathrm{V}$ - atomic system inside a doublecavity [40] is shown to be due to the leakage of population from the coherently prepared lower atomic levels. This leakage occurs via spontaneous emission into the ground state leading to instability in the lower cooperative branch. Such instability occurs only in the $\wedge$-system wherein incoherent decay coupling of the pair of ground states is essential to obtain instabilities in the cooperative branch. 
As evidenced by other articles in this volume, optical bistability is of interest

In all previous theoretical studies of OB [1-40] the usual rotating wave approximation (RWA) has been adopted, which amounts to neglecting highly oscillatory terms in the model Maxwell-Bloch equations. Effects of such terms on the bistable behavior for two-level atomic systems in an optical ring cavity were recently studied by our group for homogeneously and non-homogeneously broadened atomic medium [41-42].

It has been shown that, the first harmonic output field component, generated outside the RWA, shows reversed (clockwise) or closed (butterfly) loop bistability, simultaneously with the usual (anti-clockwise) bistable output fundamental field component. Thus, transforming opposite coding information simultaneously in such a system is a physical possibility. Also, the order of the first harmonic output field is small, $O\left(\lambda^{2}\right)\left(\lambda=\gamma / \omega_{\mathrm{L}} ; \gamma=\right.$ the atomic damping constant, $\omega_{\mathrm{L}}=$ circular frequency of the injected laser field) and sensitive to the damping ratio $(\gamma / \kappa)$; where $(\kappa)$ is the cavity damping constant. As argued in [41] such weak output signals may be detected via phase sensitive detection techniques used in detecting squeezed light. In addition, when effects of atomic linewidth are taken into consideration, the output field component outside the RWA, which exhibits the usual anti-clockwise bistable behavior in the absorptive case, switches in the dispersive case to a closed loop (switching-down process) with Lorentzian linewidth to reversed (clockwise) bistable behavior with Gaussian linewidth. 
Enhancement of the parameter $(\lambda)$ can be achieved in strong coupling regimes $(\lambda>1)$ - see recent experiments with superconductor qubits [43], solid-state semiconductors [44] and nano-mechanical resonators [45]. See also, recent theoretical investigations on entanglement of non-dissipative strongly coupled two-qubit system in a single quantized field mode outside the RWA ([46] and refs. therein), earlier studies outside the RWA on dissipative quantum systems interacting with squeezed vacuum baths [47-49] and non-dissipative mesoscopic multistable [50] and pulsed-driven qubit [51] systems. Outside the RWA, critical slowing down has been discussed recently [52] in which near the critical point of the incident field, the system exhibits switching to the lower branch of the first harmonic output field with irregular oscillations in both high and low quality cavity cases.

In the present work, we extend the results in [41] to investigate the dynamical behavior of homogeneously broadened two-level atomic systems placed in an optical ring cavity outside the RWA. We show that the presence of various types of dynamics and chaos is sensitively dependent on the atomic detuning parameter.

The paper is organized as follows: The non-autonomous model of MaxwellBloch equations outside the RWA with analytical treatment via Fourier series decomposition up to the first generated harmonic component of the output field is reviewed in Sec. 2. Nonlinear dynamics and bifurcation behavior are computationally investigated in Sec. 3. The effect of Gaussian field modestructure on the dynamical behavior is investigated in Sec. 4, followed by a summary is Sec. 5 . 


\section{Model Review}

For a single mode ring cavity containing a homogeneously broadened two-level atomic medium of length $\mathrm{L}$, the c-number model Maxwell-Bloch equations, in the plane wave and mean field approximations, and in a rotating frame at $\omega_{L}$, outside the RWA, have the dimensionless form [41]

$$
\begin{aligned}
& \frac{\mathrm{dx}}{\mathrm{dt}}=\kappa\left[\mathrm{Y}-(1+\mathrm{i} \theta) \mathrm{x}+2 \sqrt{2} \mathrm{Cr}_{-}\right], \\
& \frac{\mathrm{d}}{\mathrm{dt}} \mathrm{r}_{-}=-\frac{\gamma_{\perp}}{2}(1+\mathrm{i} \delta) \mathrm{r}_{-}+\frac{\gamma_{\perp}}{\sqrt{2}} \mathrm{r}_{3}\left(\mathrm{x}+\mathrm{x}^{*} \mathrm{e}^{\mathrm{int}}\right)=\left(\frac{\mathrm{d}}{\mathrm{dt}} \mathrm{r}_{+}\right)^{*}, \\
& \frac{\mathrm{d}}{\mathrm{dt}} \mathrm{r}_{3}=-\frac{\gamma_{11}}{2}\left(1+2 \mathrm{r}_{3}\right)-\frac{\gamma_{11}}{2 \sqrt{2}}\left[\mathrm{r}_{+}\left(\mathrm{x}+\mathrm{x}^{*} \mathrm{e}^{\mathrm{int}}\right)+\text { c.c. }\right] .
\end{aligned}
$$

The notations in equations $1 \mathrm{a}$ to $1 \mathrm{c}$ are as follows: $r_{ \pm}$are the mean values of the quadrature atomic polarization components, $r_{3}$ is the mean value of the atomic inversion, $\gamma_{\perp}$ is the transverse relaxation of the atomic polarization, $\gamma_{11}$ is the longitudinal relaxation of the atomic inversion and $\delta=2\left(\omega_{\mathrm{o}}-\omega_{\mathrm{L}}\right) / \gamma_{\perp}$ is the normalised atomic detuning, where $\omega_{0}$ is the atomic transition frequency, $\omega_{L}$ is the input field frequency, and $\eta=2 \omega_{L}$. The quantities $\mathrm{x}$ and $\mathrm{Y}$ are the normalized output and input amplitude fields, respectively, $\theta=\left(\omega_{c}-\omega_{L}\right) / \kappa$ is the normalized cavity detuning with $\omega_{c}=$ cavity mode frequency, $\kappa=$ cavity decay constant and $\mathrm{C}=\mathrm{g}^{2} /\left(\gamma_{\perp} \kappa\right)$ is the cooperative parameter, where $\mathrm{g}$ is the coupling between the cavity field and the atoms and (c.c.) stands for complex conjugate. 
The terms containing $e^{ \pm i \eta t}\left(=e^{ \pm 2 i \omega_{L} t}\right)$ in equations $(1 \mathrm{~b}, 1 \mathrm{c})$ represent the effect of interaction of the cavity field with the atoms outside the RWA. Within the RWA these terms are discarded and equations 1a-1c yield the well-known input-output field steady state equation [6-8]:

$\mathrm{Y}=\mathrm{x}_{\mathrm{o}}\left[\left(1+\frac{2 \mathrm{C}}{1+\delta^{2}+\left|\mathrm{x}_{\mathrm{o}}\right|^{2}}\right)+\mathrm{i}\left(\theta-\frac{2 \mathrm{C} \delta}{1+\delta^{2}+\left|\mathrm{x}_{\mathrm{o}}\right|^{2}}\right)\right]$,

where, $x_{o}$ is the fundamental output field amplitude within the RWA.

The solution for the atomic Bloch components $r_{\mp, 3}$ and the cavity field $\mathrm{x}$ according to equations 1a-1c contain all harmonics of frequency $2 \omega_{L} n$ ( $n=$ integer), due to the presence of the harmonic coefficients $e^{ \pm i n t}$. Following our analysis in $[41,42]$ the field and atomic variables are decomposed up to first harmonic $(n= \pm 1)$ as follows:

$r_{\mp, 3}(t)={r_{\mp, 3}}^{o}(t)+{r_{\mp, 3}}^{+}(t) e^{i \eta t}+{r_{\mp, 3}}^{-}(t) e^{-i \eta t}$

$x(t)=x_{o}(t)+x_{+}(t) e^{i \eta t}+x_{-}(t) e^{-i \eta t}$,

where, $x_{o}$ and $r_{\mp, 3}{ }^{o}$ are the fundamental field and atomic variables components, respectively, within the RWA, and the corresponding components $x_{\mp}, r_{\mp, 3}{ }^{ \pm}$are those generated outside the RWA. Substituting equation 3 into 1a-1c and comparing the coefficients of $e^{ \pm i n \eta t}(\mathrm{n}=0,1)$ we reach the following system of ordinary differential equations (ODEs) for atom and field harmonic amplitude components: 


$$
\begin{aligned}
\frac{\mathrm{d}}{\mathrm{dt}} \mathrm{r}_{-}^{\mathrm{o}}(\mathrm{t}) & =-\frac{\gamma_{\perp}}{2}(1+\mathrm{i} \delta) \mathrm{r}_{-}^{\mathrm{o}}(\mathrm{t})+\frac{\gamma_{\perp}}{\sqrt{2}}\left[\mathrm{r}_{3}^{\mathrm{o}}(\mathrm{t})\left(\mathrm{x}_{\mathrm{o}}(\mathrm{t})+\mathrm{x}_{+}^{*}(\mathrm{t})\right)+\mathrm{r}_{3}^{-}(\mathrm{t})\left(\mathrm{x}_{\mathrm{o}}^{*}(\mathrm{t})+\mathrm{x}_{+}(\mathrm{t})\right)+\mathrm{r}_{3}^{+}(\mathrm{t}) \mathrm{x}_{-}(\mathrm{t})\right] \\
& =\left(\frac{d}{d t} r_{+}^{o}(t)\right)^{*}
\end{aligned}
$$$$
\frac{\mathrm{d}}{\mathrm{dt}} \mathrm{r}_{-}^{+}(\mathrm{t})=-\frac{\gamma_{\perp}}{2}\left(1+\mathrm{i} \delta+\frac{2 \mathrm{i} \eta}{\gamma}\right) \mathrm{r}_{-}^{+}(\mathrm{t})+\frac{\gamma_{\perp}}{\sqrt{2}}\left[\mathrm{r}_{3}^{\mathrm{o}}(\mathrm{t})\left(\mathrm{x}_{\mathrm{o}}^{*}(\mathrm{t})+\mathrm{x}_{+}(\mathrm{t})\right)+\mathrm{r}_{3}^{+}(\mathrm{t})\left(\mathrm{x}_{\mathrm{o}}(\mathrm{t})+\mathrm{x}_{+}^{*}(\mathrm{t})\right)\right.
$$$$
\left.+\mathrm{r}_{3}^{-}(\mathrm{t}) \mathrm{x}_{-}^{*}(\mathrm{t})\right]
$$$$
=\left(\frac{d}{d t} r_{+}^{-}(t)\right)^{*},
$$$$
\frac{\mathrm{d}}{\mathrm{dt}} \mathrm{r}_{-}^{-}(\mathrm{t})=-\frac{\gamma_{\perp}}{2}\left(1+\mathrm{i} \delta-\frac{2 \mathrm{i \eta}}{\gamma}\right) \mathrm{r}_{-}^{-}(\mathrm{t})+\frac{\gamma_{\perp}}{\sqrt{2}}\left[\mathrm{r}_{3}^{\mathrm{o}}(\mathrm{t}) \mathrm{x}_{-}(\mathrm{t})+\mathrm{r}_{3}^{-}(\mathrm{t})\left(\mathrm{x}_{\mathrm{o}}(\mathrm{t})+\mathrm{x}_{+}^{*}(\mathrm{t})\right)\right]
$$$$
=\left(\frac{d}{d t} r_{+}^{+}(t)\right)^{*}
$$$$
\frac{\mathrm{d}}{\mathrm{dt}} \mathrm{r}_{3}^{\mathrm{o}}(\mathrm{t})=-\frac{\gamma_{11}}{2}-\gamma \mathrm{r}_{3}^{\mathrm{o}}(\mathrm{t})-\frac{\gamma_{11}}{2 \sqrt{2}}\left[\left(\mathrm{r}_{+}^{\mathrm{o}}(\mathrm{t})+\mathrm{r}_{-}^{+}(\mathrm{t})\right)\left(\mathrm{x}_{\mathrm{o}}(\mathrm{t})+\mathrm{x}_{+}^{*}(\mathrm{t})\right)+\mathrm{r}_{+}^{+}(\mathrm{t}) \mathrm{x}_{-}(\mathrm{t})+\text { c.c.. }\right] \text {, }
$$$$
\frac{\mathrm{d}}{\mathrm{dt}} \mathrm{r}_{3}^{+}(\mathrm{t})=-\gamma_{11}\left(1+\frac{\mathrm{i} \eta}{\gamma}\right) \mathrm{r}_{3}^{+}(\mathrm{t})-\frac{\gamma_{11}}{2 \sqrt{2}}\left[\left(\mathrm{r}_{+}^{\mathrm{o}}(\mathrm{t})+\mathrm{r}_{-}^{+}(\mathrm{t})\right)\left(\mathrm{x}_{\mathrm{o}}^{*}(\mathrm{t})+\mathrm{x}_{+}(\mathrm{t})\right)+\mathrm{r}_{+}^{+}(\mathrm{t})\left(\mathrm{x}_{\mathrm{o}}(\mathrm{t})+\mathrm{x}_{+}^{*}(\mathrm{t})\right)\right.
$$$$
\left.+\mathrm{r}_{+}^{-}(\mathrm{t}) \mathrm{x}_{-}^{*}(\mathrm{t})+\mathrm{r}_{-}^{\mathrm{o}}(\mathrm{t}) \mathrm{x}_{-}^{*}(\mathrm{t})\right]
$$$$
=\left(\frac{d}{d t} r_{3}^{-}(t)\right)^{*},
$$

$$
\frac{\mathrm{dx}_{\mathrm{o}}}{\mathrm{dt}}=\kappa\left[\mathrm{Y}-(1+\mathrm{i} \theta) \mathrm{x}_{\mathrm{o}}(\mathrm{t})+2 \sqrt{2} \mathrm{C} \mathrm{r}_{-}^{\mathrm{o}}(\mathrm{t})\right] \text {, }
$$

$$
\frac{\mathrm{dx}_{+}}{\mathrm{dt}}=-\kappa\left[\left(1+\mathrm{i} \theta+\frac{\mathrm{i \eta}}{\kappa}\right) \mathrm{x}_{+}(\mathrm{t})-2 \sqrt{2} \mathrm{Cr}_{-}^{+}(\mathrm{t})\right],
$$

$$
\frac{\mathrm{dx}_{-}}{\mathrm{dt}}=-\kappa\left[\left(1+\mathrm{i} \theta-\frac{\mathrm{i \eta}}{\kappa}\right) \mathrm{x}_{-}(\mathrm{t})-2 \sqrt{2} \mathrm{C} \mathrm{r}_{-}^{-}(\mathrm{t})\right] \text {. }
$$


Our analytical and numerical results $[41,42]$ show that the amplitude of the additional first harmonic component $x_{+}$, is of smaller order, $O\left(\lambda^{2}\right)$ with $\lambda=\gamma / 2 \omega_{\mathrm{L}}$ , compared with the fundamental field component amplitude $\mathrm{x}_{\mathrm{o}}$, and to the same order of approximation the field component $x_{-}=0$ and hence the dipole component $r_{-}^{-}=0$. Consequently, equations $4 \mathrm{a}-4 \mathrm{~h}$ reduce to the following smaller set of ODEs:

$$
\begin{aligned}
\frac{\mathrm{d}}{\mathrm{dt}} \mathrm{r}_{-}^{\mathrm{o}}(\mathrm{t}) & =-\frac{\gamma_{\perp}}{2}(1+\mathrm{i} \delta) \mathrm{r}_{-}^{\mathrm{o}}(\mathrm{t})+\frac{\gamma_{\perp}}{\sqrt{2}} \mathrm{r}_{3}^{\mathrm{o}}(\mathrm{t}) \mathrm{x}_{\mathbf{o}}(\mathrm{t}) \\
& =\left(\frac{\mathrm{d}}{\mathrm{dt}} \mathrm{r}_{+}^{\mathrm{o}}(\mathrm{t})\right)^{*},
\end{aligned}
$$

$$
\frac{\mathrm{d}}{\mathrm{dt}} \mathrm{r}_{-}^{+}(\mathrm{t})=-\frac{\gamma_{\perp}}{2}\left(1+\mathrm{i} \delta+\frac{2 \mathrm{i \eta}}{\gamma_{\perp}}\right) \mathrm{r}_{-}^{+}(\mathrm{t})+\frac{\gamma_{\perp}}{\sqrt{2}} \mathrm{r}_{3}^{\mathrm{o}}(\mathrm{t}) \mathrm{x}_{\mathrm{o}}^{*}(\mathrm{t})
$$

$$
=\left(\frac{\mathrm{d}}{\mathrm{dt}} \mathrm{r}_{+}^{-}(\mathrm{t})\right)^{*},
$$

$$
\frac{\mathrm{d}}{\mathrm{dt}} \mathrm{r}_{3}^{\mathrm{o}}(\mathrm{t})=-\frac{\gamma_{11}}{2}-\gamma_{11} \mathrm{r}_{3}^{\mathrm{o}}(\mathrm{t})-\frac{\gamma_{11}}{2 \sqrt{2}}\left[\mathrm{r}_{+}^{\mathrm{o}}(\mathrm{t}) \mathrm{x}_{\mathrm{o}}(\mathrm{t})+\mathrm{c.c} .\right]
$$

$\frac{\mathrm{dx}_{\mathrm{o}}}{\mathrm{dt}}=\kappa\left[\mathrm{Y}-(1+\mathrm{i} \theta) \mathrm{x}_{\mathrm{o}}(\mathrm{t})+2 \sqrt{2} \mathrm{C} \mathrm{r}_{-}^{\mathrm{o}}(\mathrm{t})\right]$

$$
\frac{\mathrm{dx}_{+}}{\mathrm{dt}}=-\kappa\left[\left(1+\mathrm{i} \theta+\frac{\mathrm{i \eta}}{\kappa}\right) \mathrm{x}_{+}(\mathrm{t})-2 \sqrt{2} \mathrm{C} \mathrm{r}_{-}^{+}(\mathrm{t})\right] \text {. }
$$

Traditionally optical instabilities may arise in two well known regimes [25, 53]:

(i) Interaction of the atomic system with (large) multimode field that results from the interplay of varity of time scales leading to chaos and self-pulsing. 
(ii) Interaction of the atomic system with the intense single cavity mode that saturates the collection of atoms in the absorptive OB which does not involve any cooperative effects.

It is well known that when the system is in the unstable region of the upper branch of the OB curve, either it precipitates to the lower transmission branch or exhibits oscillatory behavior which is either periodic (self-pulsing) or non-periodic (chaotic). The analytical study of instability [11] for absorptive and dispersive bistability shows that the instability of the upper branch may exist if at least one of the off-resonant modes are unstable. Now we investigate the nonlinear dynamical behavior of the OB system within and outside the RWA in the dispersive case when the atomic polarization variables are eliminated adiabatically. In this adiabatic case, the atomic relaxation time for the atomic polarization components is shorter than the other characteristic times ( $\left.\left.\left.\gamma_{\perp}\right\rangle\right\rangle \gamma_{l l}, \kappa, \kappa \theta, \kappa C\right)$ of the system. Hence by substuting in equations $5 \mathrm{a}-5 \mathrm{e}$ for $\mathrm{r}_{ \pm}^{\mathrm{o}, \pm}$ by their stationary values [41], and by expressing the normalized output fields, $\mathrm{x}_{\mathrm{o}}=\mathrm{u}_{\mathrm{o}}+\mathrm{iv}_{\mathrm{o}}$ and $\mathrm{x}_{+}=\mathrm{u}_{+}+\mathrm{iv}_{+}$, we obtain the following system of ODEs:

$$
\begin{aligned}
& \kappa^{\prime} \frac{\mathrm{dr}_{3}^{\mathrm{o}}(\tau)}{\mathrm{d} \tau}=-\frac{1}{2}\left(1+2 \mathrm{r}_{3}^{\mathrm{o}}(\tau)\right)-\frac{\mathrm{r}_{3}^{\mathrm{o}}(\tau)}{\left(1+\delta^{2}\right)}\left(\mathrm{u}_{\mathrm{o}}^{2}(\tau)+\mathrm{v}_{\mathrm{o}}^{2}(\tau)\right), \\
& \frac{\mathrm{du}_{\mathrm{o}}}{\mathrm{d} \tau}=\mathrm{Y}-\mathrm{u}_{\mathrm{o}}+\theta \mathrm{v}_{\mathrm{o}}+4 \mathrm{C} \mathrm{r}_{3}^{\mathrm{o}} \frac{\left(\delta \mathrm{v}_{\mathrm{o}}+\mathrm{u}_{\mathrm{o}}\right)}{1+\delta^{2}} \\
& \frac{\mathrm{dv}_{\mathrm{o}}}{\mathrm{d} \tau}=-\mathrm{v}_{\mathrm{o}}-\theta \mathrm{u}_{\mathrm{o}}+4 C \mathrm{r}_{3}^{\mathrm{o}} \frac{\left(-\delta \mathrm{u}_{\mathrm{o}}+\mathrm{v}_{\mathrm{o}}\right)}{1+\delta^{2}}
\end{aligned}
$$




$$
\begin{aligned}
& \frac{d u_{+}}{d \tau}=-u_{+}+\left(\theta+\frac{1}{v}\right) v_{+}-4 C r_{3}^{o} \frac{\left(\left(\delta+\frac{2}{\lambda}\right) v_{o}-u_{o}\right)}{1+\left(\delta+\frac{2}{\lambda}\right)^{2}} \\
& \frac{d v_{+}}{d \tau}=-v_{+}-\left(\theta+\frac{1}{v}\right) u_{+}-4 C r_{3}^{o} \frac{\left(\left(\delta+\frac{2}{\lambda}\right) u_{o}+v_{o}\right)}{1+\left(\delta+\frac{2}{\lambda}\right)^{2}}
\end{aligned}
$$

with $\tau=\gamma_{\perp} \mathrm{t}, \kappa^{\prime}=\kappa / \gamma_{\perp}, \lambda=\gamma / \eta$ and $v=\kappa / \eta$.

Equations $6 \mathrm{a}-6 \mathrm{e}$ are treated numerically in the next section using the standard Runge-Kutta method with suitable initial conditions to display various dynamical states. For the absorbing medium we take $r_{3}^{\circ}(0)=-0.5$ with cavity fields $x_{0,+}(0)=0$

\section{Computational Results}

Nonlinear systems that display chaos are sensitive to the system parameters and essentially show four modes of operations: chaotic, stable, periodic and quasiperiodic. This can be demonstrated in the bifurcation diagram for the normalized output field components within and outside the RWA $\left(\left|x_{0}\right|\right.$ and $\left.\left|x_{+}\right|\right)$ against the input field $\mathrm{Y}$ for fixed system parameters $(\delta=374, \theta=340, \mathrm{C}=70000$ $\left., \lambda=10^{-6}, \bar{\kappa}=0.25\right)$ as seen in Fig. (1a,b). It is noted that, optical bistable devices can display chaotic behavior, multi-periodic and single periodic states for both output field components by increasing the input field Y. In other words, the OB device routes from chaos to self pulsing by increasing Y. In particular, chaotic behavior occurs at $\mathrm{Y}=950$, and is transformed to 4-periods doubling at $\mathrm{Y}=1225$, to 2-periods at $\mathrm{Y}=1350$ and finally to a single period at $\mathrm{Y}=2000$. These results 
were obtained earlier in [53] for the output field component $\left|x_{0}\right|$ within the RWA. Note that, each point in the bifurcation diagram represents the peak of the oscillation.

The steady state solution that represents the relation between the output field component outside the RWA, $\left|\mathrm{x}_{+}\right|$and the input field $\mathrm{Y}$ in the dispersive case for different detuning parameters $(\delta, \theta)$ is shown in Figure $(2 \mathrm{a}, \mathrm{b})$. The variation of the detuning parameters leads to closed loop behavior [41]. Linear stability analysis around the stationary state shows that, points on the upper branch of the bistable behavior are unstable and the associated instability arises with opposite signs of the detuning parameters ( $\delta \theta<0)$ and multiple periods of unstable points occurs for large cooperativity (C>300) [25]. In the (reversed) bistable behavior over a certain input range, $Y \in(0,2500)$, as shown in the inset of Fig. $(2 b)$, the points A, B, C, D represent the unstable points in the upper branch of Fig. (2b) and correspond to the respective $\mathrm{Y}$ values as $\mathrm{Y}=950, \mathrm{Y}=1225, \mathrm{Y}=1350$ and $\mathrm{Y}=2000$, respectively.

The system of normalized coupled equations $6 \mathrm{a}-6 \mathrm{e}$ is integrated numerically for fixed values of $\theta=340, \delta=374, \bar{\kappa}=0.25$. For different $Y$ values, the timedependence of the output field components $\left|\mathrm{x}_{\mathrm{o}}\right|$ and $\left|x_{+}\right|$, the phase portrait ( $\left.\operatorname{Im}\left(\left|\mathrm{x}_{\mathrm{o},+}\right|\right), \operatorname{Re}\left(\mathrm{x}_{\mathrm{o},+} \mid\right)\right), 2-\mathrm{D}$ Poincare map of the solutions at fixed $\mathrm{r}_{3}^{\mathrm{o}}$ and power spectra diagrams of the input intensities are plotted respectively in Figs. (3-6) for fixed parameters of the system. For $\mathrm{Y}=2000$ (Fig. 3a,b), periodic behavior is displayed for both field components $\left(\left|\mathrm{x}_{\mathrm{o}}\right|,\left|x_{+}\right|\right)$but with significant spikes for the output field component of the RWA $\left|x_{+}\right|$. A single period is exhibited in the phase 
space of the output field component $\left|\mathrm{x}_{\mathrm{o}}\right|$, but with weak oscillations occuring in the single period of the $\left|x_{+}\right|$component (Fig. 3b). The presence of spikes in the phase portrait is due to the oscillatory nature of the systems that operate outside the RWA that generate the virtual photons. This single periodic loop appears in the Poincare diagram of the output field components $\left(\left|\mathrm{x}_{\mathrm{o}}\right|,\left|x_{+}\right|\right)$as a single point (Fig. 3a, b). The power spectrum shows more oscillations for the component $\left|x_{+}\right|$ outside the RWA, as compared with the component $\left|x_{0}\right|$ within the RWA.

Decreasing $\mathrm{Y}$ to $\mathrm{Y}=1350$ (Fig. 4a,b), double periods occur for both field components $\left|\mathrm{x}_{\mathrm{o}}\right|$ and $\left|x_{+}\right|$, but with weak oscillatory behavior in the $\left|x_{+}\right|$-phase portrait (Fig. 4b). The Poincaré map that confirms the existence of period doubling is shown by two points. At $Y=1225$ we have four periods (Fig. 5) and finaly at $\mathrm{Y}=950$ the chaotic behavior is exhibited (Fig.6). Notice the random behavior for the field component $\left|x_{+}\right|$as seen in the phase space of Figs. $(5 \mathrm{~b}, 6 \mathrm{~b})$. For fixed $Y=1350$, the system can be converted from chaotic to aperiodic behavior for both components $\left|\mathrm{x}_{\mathrm{o}}\right|,\left|x_{+}\right|$by increasing the atomic detuning from $\delta=360$ to 374 or vice-versa (Fig. 7).

\section{Transverse Field Effect}

Here, we consider that the field and atomic variables are allowed to vary along the radial direction by taking into account the possible variations of the field along the transverse directions, hence, we can study the effect of the transverse field on the dynamics of the OB device. The reduced Maxwell equations, within the one transverse mode approximation for the unidirectional normalised field 
component in the ring cavity configuration, together with the Bloch equations for homogeneously broadened 2-level atomic systems driven by a Gaussian field $\sqrt{\mathrm{a}(\rho)} \mathrm{x}(\mathrm{t})$ within the mean field approximation are [42]:

$$
\begin{aligned}
& \frac{\partial}{\partial t} \mathbf{r}_{-}^{\mathrm{o}}(\mathrm{t})=-\frac{\gamma}{2}(1+\mathrm{i} \delta) \mathrm{r}_{-}^{\mathrm{o}}(\mathrm{t})+\frac{\gamma}{\sqrt{2}} \sqrt{\mathrm{a}(\rho) \mathrm{r}_{3}^{\mathrm{o}}}(\mathrm{t}) \mathrm{x}_{\mathrm{o}}(\mathrm{t}) \\
& =\left(\frac{\partial}{\partial \mathrm{t}} \mathrm{r}_{+}^{\mathrm{o}}(\mathrm{t})\right)^{*}, \\
& \frac{\partial}{\partial t} r_{-}^{+}(t)=-\frac{\gamma}{2}\left(1+i \delta+\frac{2 i \eta}{\gamma}\right) r_{-}^{+}(t)+\sqrt{a(\rho)} r_{3}^{o}(t) x_{o}^{*}(t) \\
& =\left(\frac{\partial}{\partial \mathrm{t}} \mathrm{r}_{+}^{-}(\mathrm{t})\right)^{*} \\
& \frac{\partial}{\partial t} r_{3}^{o}(t)=-\frac{\gamma}{2}-\gamma r_{3}^{o}(t)-\frac{\gamma \sqrt{a(\rho)}}{2 \sqrt{2}}\left[r_{+}^{o}(t)\left(x_{o}(t)+r_{-}^{o}(t) x_{o}^{*}(t)\right],\right. \\
& \frac{d x_{o}}{d t}=\kappa\left[Y-(1+i \theta) x_{o}(t)+2 \sqrt{2} C \int_{h}^{1} a(\rho)^{-\frac{1}{2}} r_{-}^{o}(t) d a(\rho)\right] \text {, } \\
& \frac{\mathrm{dx}_{+}}{\mathrm{dt}}=-\kappa\left[\left(1+\mathrm{i} \theta+\frac{\mathrm{i} \eta}{\kappa}\right) \mathrm{x}_{+}(\mathrm{t})-2 \sqrt{2} \mathrm{C} \int_{\mathrm{h}}^{1} \mathrm{a}(\rho)^{-\frac{1}{2}} \mathrm{r}_{-}^{+}(\mathrm{t}) \mathrm{da}(\rho)\right] \text {, }
\end{aligned}
$$

Here $a(\rho)=e^{-\left(\frac{2 \rho^{2}}{w_{o}}\right)}$ is the Gaussian field shape for Fresnel number $F>>1, h=a\left(\rho_{0}\right)$ , in which $\rho_{0}$ is the radius of the atomic sample and $w_{o}$ is the spot size or beam waist. In the case of adiatatic elimination of atomic polarization, equations 7a7 e reduce to the following system of ODEs for the output field components $\mathrm{x}_{\mathrm{o}}=\mathrm{u}_{\mathrm{o}}+\mathrm{v}_{\mathrm{o}}$ and $\mathrm{x}_{+}=\mathrm{u}_{+}+\mathrm{v}_{+}$, 


$$
\begin{aligned}
& \frac{d u_{o}}{d \tau}=Y-u_{o}(\tau)+\theta v_{o}(\tau)+\frac{4 C}{\left(1+\delta^{2}\right)} \int_{h}^{1}\left(u_{o}(\tau)+\delta v_{o}(\tau)\right) r_{3}^{o}(\tau) d a \\
& \frac{d v_{o}}{d \tau}=-v_{o}(\tau)-\theta u_{o}(\tau)+\frac{4 C}{\left(1+\delta^{2}\right)} \int_{h}^{1}\left(v_{o}(\tau)-\delta u_{o}(\tau)\right) r_{3}^{o}(\tau) d a \\
& \frac{d u_{+}}{d \tau}=-u_{+}(\tau)+\left(\theta+\frac{1}{v}\right) v_{+}(\tau)+\frac{4 C}{\left(1+\left(\delta+\frac{2}{\lambda}\right)^{2}\right)} \int_{h}^{1}\left(u_{o}(\tau)-\left(\delta+\frac{2}{\lambda}\right) v_{o}(\tau)\right) r_{3}^{o}(\tau) d a \\
& \frac{d v_{+}}{d \tau}=-v_{+}(\tau)-\left(\theta+\frac{1}{v}\right) u_{+}(\tau)-\frac{4 C}{\left(1+\left(\delta+\frac{2}{\lambda}\right)^{2}\right)^{h}}\left(v_{o}(\tau)+\left(\delta+\frac{2}{\lambda}\right) u_{o}(\tau)\right) r_{3}^{o}(\tau) d a \\
& \frac{\partial}{\partial \tau} r_{3}^{o}(\tau)=\frac{1}{\bar{\kappa}}\left(-\frac{1}{2}-r_{3}^{o}(\tau)-a_{3}^{o}(\tau) \frac{v_{o}^{2}(\tau)+u_{o}^{2}(\tau)}{\left(1+\delta^{2}\right)}\right)
\end{aligned}
$$

For large detuning $(\delta>>1)$, as we consider throughout this paper, equation $8 \mathrm{e}$ can be reduced to (see App. I)

$$
\frac{\partial}{\partial \tau} \mathbf{r}_{3}^{\mathrm{o}}(\tau)=\frac{1}{\bar{\kappa}}\left(-\frac{1}{2}-\mathrm{r}_{3}^{\mathrm{o}}(\tau)\right)
$$

Hence, $r_{3}^{\circ}(\tau)$ becomes independent of the transverse field parameter and so equations $8 \mathrm{a}-8 \mathrm{e}$ become

$$
\begin{aligned}
& \frac{\mathrm{du}_{\mathrm{o}}}{\mathrm{d} \tau}=\mathrm{Y}-\mathrm{u}_{\mathrm{o}}(\tau)+\theta \mathrm{v}_{\mathrm{o}}(\tau)+\frac{4 \mathrm{C}}{\left(1+\delta^{2}\right)}\left(\mathrm{u}_{\mathrm{o}}(\tau)+\delta \mathrm{v}_{\mathrm{o}}(\tau)\right) \mathrm{r}_{3}^{\mathrm{o}}(\tau)(1-\mathrm{h}) \\
& \frac{\mathrm{dv_{ \textrm {o } }}}{\mathrm{d} \tau}=-\mathrm{v}_{\mathrm{o}}(\tau)-\theta \mathrm{u}_{\mathrm{o}}(\tau)+\frac{4 \mathrm{C}}{\left(1+\delta^{2}\right)}\left(\mathrm{v}_{\mathrm{o}}(\tau)-\delta \mathrm{u}_{\mathrm{o}}(\tau)\right) \mathrm{r}_{3}^{\mathrm{o}}(\tau)(1-\mathrm{h}) \\
& \frac{\mathrm{du} \mathrm{u}_{+}}{\mathrm{d} \tau}=-\mathrm{u}_{+}(\tau)+\left(\theta+\frac{1}{v}\right) \mathrm{v}_{+}(\tau)+\frac{4 C}{\left(1+\left(\delta+\frac{2}{\lambda}\right)^{2}\right)}\left(\mathrm{u}_{\mathrm{o}}(\tau)-\left(\delta+\frac{2}{\lambda}\right) \mathrm{v}_{\mathrm{o}}(\tau)\right) \mathrm{r}_{3}^{\mathrm{o}}(\tau)(1-\mathrm{h})
\end{aligned}
$$


$\frac{d v_{+}}{d \tau}=-v_{+}(\tau)-\left(\theta+\frac{1}{v}\right) u_{+}(\tau)-\frac{4 C}{\left(1+\left(\delta+\frac{2}{\lambda}\right)^{2}\right)}\left(v_{o}(\tau)+\left(\delta+\frac{2}{\lambda}\right) u_{o}(\tau)\right) r_{3}^{o}(\tau)(1-h)$,

where $r_{3}^{\circ}(\tau)$ obeys equation 9 .

\section{Summary}

We have investigated the dynamical behavior of an optical bistable system for homogeneously broadened two-level atoms interacting with a single mode ring cavity outside the RWA in the dispersive regime. The system routes from periodic to chaotic behavior by decreasing the input field. This can be viewed from the bifurcation diagram $\left(\left|x_{0,+}\right|\right.$ against $\left.Y\right)$ by sweeping the parameter $Y$ forth and back. Outside the RWA, the virtual photons (that results from rapidly oscillating terms of the atom-field interaction) affect the self-pulsing and chaotic behaviors of the unstable state of the system in the form of spiking. The state of the OB device changing from regular (self-pulsing) to irregular (chaos) oscillations by varying the atomic detuning as seen in the bifurcation diagram of the output field against the atomic detuning. Gaussian field mode shape has the effect to

\section{References}

[1] A. Szöke, V. Daneu, J. Goldhar and N. A. Kurnit, Appl. Phys. Lett.15 (1969) 376.

[2] S. L. McCall, Phys. Rev. A $\underline{9}$ (1974) 1515.

[3] H. M. Gibbs, S. L. McCall and T. N. C. Venkatesan, Phys. Rev. Lett. $\underline{\mathbf{3 6}}$ (1976) 1135. 
[4] H. M. Gibbs, S. L. McCall and T. N. C. Venkatesan, Opt. News $\underline{5}$ (1979) 6.

[5] R. Bonifacio and L. A. Lugiato, Optics Commun. $\underline{19}$ (1976) 172 .

[6] R. Bonifacio and L. A. Lugiato, Phys. Rev. A $\underline{18}$ (1978) 1129

[7] R. Bonifacio and L. A. Lugiato, Lett. Nuovo Cimento 21 (1978) 517.

[8] S. S. Hassan, P. D. Drummond and D. F. Walls, Optics Commun. 27 (1978) 480.

[9] E. Abraham, R. K. Bullough and S. S. Hassan, Optics Commun. 29 (1979) 109; E. Abraham, S. S. Hassan and R. K. Bullough, Optics Commun. $\underline{33}$ (1980) 93; E. Abraham and S. S. Hassan, Optics Commun. $\underline{35}$ (1980) 291.

[10] E. Abraham and S. D. Smith, J. Phys. E 15 (1982) 33; E. Abraham and S. D. Smith, Rep. Prog. Phys. $\underline{45}$ (1982) 815.

[11] L. A. Lugiato, in: E. Wolf (Ed.) Progress in Optics, Vol. 21 (North Holland, Amsterdam, 1984), pp.71-183; L. A. Lugiato and L. M. Narducci, in: J. Dalibard, J. M. Raimond and J. Zinn-Justin (Eds.), Fundamental Systems in Quantum Optics (Elsevier Sci. Publ., Amsterdam, 1992), PP 942-1043.

[12] B. S. Wherrett and P. Chavel (Eds.), Optical Computing, (Inst. of Physics Publ., Bristol, 1995); J. N. Lee (Ed.), Design Issues in Optical Processing (Cambridge Univ. Press, Cambridge, UK, 1995); D. G. Feitelson, Optical Computing (MIT Press. MA., 1988).

[13] C. M. Bowden, M. Ciftan and H. R. Robl (Eds), 'Optical Bistability' (Plenum Press, NY,1981).

[14] L. A. Lugiato, V. Benza, and L. M. Narducci, J. D. Farina, Opt. Commun. 39, 405 (1981).

[15] A. Joshi, and M. Xiao Appl. Phys. B, $\underline{79}, 65$ (2004).

[16] M. J. Adams, A. Hurtado, D. Labukhin and I. D. Henning, Choas $\underline{20}$ (2012) 037102

[17] Joshi, Amitabh and Xiao, Min, J. Mod. Opt. 57,1196 (2010).

[18] D. J. Gauthier, Science 279, 1156 (1998); G. D. Van Wiggeren, R. Roy, Science 279, 1198 (1998)

[19] J. T. Gteeson, Appl. Phys. Lett. 81, 1949 (2002); T. Stojanvoski, J. Pihl and L. Kocarev, IEEE. Trans. Circuits Syst. I $\underline{48}, 382$ (2001).

[20] S. L. McCall, Appl. Phys. Lett. 320, 284 (1974)].

[21] R. Bonifacio, M. Gronchi, L.A. Lugiato, Opt. Commun.30, 129 (1979).

[22] L.A. Lugiato, Opt. Commun. $\underline{\mathbf{3 3}}, 108$ (1980). 
[23] K. Ikeda, H. Daido, and O. Akimato, Phys. Rev. Lett., 45, 709 (1980); K. Ikeda and O. Akimoto, Phys. Rev. Lett. $\underline{\mathbf{4 8}}, 617$ (1982); K. Ikeda, Opt. Commun. $\underline{\text { 30 }} 257$ (1979).

[24] H. Haken, Phys. Lett.A $\underline{\mathbf{5 3}}, 77$ (1975).

[25] L. A. Orozco, H. J. Kimble, A. T. Rosenberger, L. A. Lugiato, M. L. Asquini, M. Brambilla and L. M. Narducci, Phys. Rev. A $\underline{\mathbf{3 9}}, 1235$ (1989).

[26] S. M. A. Maize, Nonlinear Optics: Quant. Opt. 35, 331 (2006); S. M. A. Maize and G.Ibrahim, ibid 35, 285 (2006); S. M. A. Maize, G. Ibrahim and M. F. M. Ali, Nonlinear Optics: Quant. Opt. 36, 117 (2007).

[27] H. A. Batrafi, S. S. Hassan, R. Saunders and R. K. Bullough, Eur. Phys. J. D $\underline{\mathbf{8}}(2000), 417$.

[28] F. Prati, L. Lugiato, Eur. Phys. J. Special Topics 203, 2012, 117.

[29] C. M. Savage, H. J. Carmichael, and D. F. Walls, Opt. Commun. 42, 3 (1982).

[30] P. Grangier, J. F. Roch, and J. Roger, L. A. Lugiato, E. M. Pessina, and G. Scandroglio, P. Galatola, Phys. Rev. A $\underline{\mathbf{4 6}}, 2735$ (1992).

[31] Wenge Yang, Amitabh Joshi, and Min Xiao Phys. Rev. Lett. 95, 093902 (2005).

[32] S. M. A. Maize, G. Ibrahim and Y. A. Sharaby Nonlinear Optics, Quantum Optics, $\underline{\mathbf{3 8}}, 2008,67$.

[33] B. V. Thompson, J. A. Hermann, Phys. Lett. A $\underline{\mathbf{8 3}}, 1981376$.

[34] S. M. A. Maize, Chaos, Solitons and Fractals 28 $2006,590$.

[35] H.A. Babu, and H. Wanare, Phys. Rev. A, $\underline{\mathbf{8 3}}, 033819$ (2011).

[36] A. Lambrecht, E. Giacobino, and J. M. Courty, Opt. Commun. 115199 (1995).

[37] H. Wang, D. J. Goorskey, and M. Xiao, Phys. Rev. A $\underline{65} 011801$ (2001); W. Yang, A. Joshi, and M. Xiao, Phys. Rev. A 므, 033807 (2004).

[38] H. M. Gibbs, F. A. Hopf, D. L. Kaplan, and R. L. Shoemaker, Phys. Rev. Lett. $\underline{\mathbf{4 6}}, 474$ (1981).

[39] H. J. Carmichael, Phy. Rev. Lett. 521292 (1984).

[40] H. Aswath Babu and Harshawardhan Wanare, arXiv:1302.1694v1 [physics.optics], 2013.

[41] Y. A. Sharaby, A. Joshi and S. S. Hassan, Phys. Letts. A $\underline{\mathbf{3 7 4}}$, (2010) 2188. [42] S. S. Hassan, Y. A. Sharaby, M. F. Ali and A. Joshi, Phys. Lett. A $\underline{\mathbf{3 7 6}}, 3555$ (2012). 
[43] J. Clarke and F. K. Wilhelm, Nature $\underline{453}$ (2008) 1031.

[44] G. Günter1, A. A. Anappara, H. Hees, A. Sell, G. Biasiol, L. Sorba, S. De. Liberato, C. Ciuti, A. Tredicucci, A. Leitenstorfer and R. Huber, Nature $\underline{458}$ (2009) 178.

[45] A. Wallraff, D. I. Schuster, A. Blais, L. Frunzio, R. S. Huang, j. Majer, S. Kumar, S. M. Girvin and R. J. Schoelkopf, Nature 431 (2004) 162.

[46] J. Song, Y. Xia, X. D, Sun, Y. Zhang, B. Liu and H. S. Song, Eur. Phys. J. D $\underline{66}$ (2012) 90 .

[47] S. S. Hassan, H. A. Batarfi and R. K. Bullough, J. Opt. B: Quantum Semiclass. Opt. $\underline{\mathbf{2}}$ (2000) R35.

[48] S. S. Hassan and O. M. Frege, J. Opt. B: Quantum Semiclass. Opt. 4 (2004) S218.

[49] S. S. Hassan, A. Joshi, O. M. Frege, and W. Emam, Ann. of Phys. $\underline{\mathbf{3 2 2}}$ (2007) 2007.

[50] S. S. Hassan and Y. A. Sharaby, J. Phys. B: At. Mol. Opt. Phys. 41 (2008) 175502; H. A. Batarfi, Y. A. Sharaby and S. S. Hassan, J. Nonlinear Opt. Phys. \&Materials 21, 1250043 (2012).

[51] S. S. Hassan, A. Joshi and N. M. Madhari, J. Phys. B 41 (2008) 145503; J. Phys. B $\underline{42}$ (2009) 089801 (Corrigendum).

[52] Y. A. Sharaby, S. S. Hassan and A. Joshi, 2013, submitted.

[53] L. A. Lugiato, L. M. Narducci, D. K. Bandy, C. A. Pennise Opt. Comm. 43, 281 (1982).

[54] B. Segard, B. Macke, L.A. Lugiato, F. Prati and M. Brambilla, Phys. Rev. A 39, 703 (1989).

\section{Figure Captions}

Fig. 1: (a) Bifurcation diagram represents the maximum value of the output field within RWA against the input field $\mathrm{Y}$ for fixed $\mathrm{C}=70000, \delta=374, \theta=340$

(b) As (a) but for the first harmonic output field component $\left|x_{+}\right|$for $\bar{\kappa}=0.25 \lambda=10^{-6}$. 
Fig. 2: (a) The first harmonic output field component $\left|x_{+}\right|$against the incident field $\mathrm{Y}$ for $\mathrm{C}=70000, \bar{\kappa}=0.25 \lambda=10^{-6}$ and different values of detuning parameters $\delta=30, \theta=50(-), \delta=100, \theta=120(\cdots \cdots \cdots)$ and $\delta=220$ ,$\theta=225(-\cdot-\cdot)$.

(b) Same as (a) but for $\delta=374, \theta=340(---)$. Inset shows the zooming of $\left|x_{+}\right|$for $Y \in[0,2500]$.

Fig. 3: (a) Self pulse oscillations for both $\left|x_{o}\right|$ and $\left|x_{+}\right|$components in the adiabatic case against the time $\tau=k t$ for $\mathrm{C}=70000, \bar{\kappa}=0.25, \lambda=10^{-6}$, $\delta=374, \theta=340$ and $\mathrm{Y}=2000$.

(b) The corresponding phase-space representation on $\left(\operatorname{Re}\left(x_{o}\right) \operatorname{Im}\left(x_{o}\right)\right)$ for the fundamental output field component and on $\left(\operatorname{Re}\left(x_{+}\right) \operatorname{Im}\left(x_{+}\right)\right)$for the first harmonic output field component respectively.

Poincare map for both $\left|\mathrm{x}_{\mathrm{o}}\right|$ and $\left|\mathrm{x}_{+}\right|$.

Fig.4: Same as (2) but with $Y=1350$.

Fig. 5: Same as (2) but with $Y=1225$.

Fig. 6: Same as (2) but with $Y=950$.

Fig. 7: (a) Bifurcation diagram that represents the maximum value of the output field within RWA against the atomic detuning $\delta$ for fixed values of other parameters as in Fig. 1, but with $\mathrm{Y}=1350$. Insets are phase portrait of $\operatorname{Re}\left(x_{o}\right) \operatorname{Im}\left(x_{o}\right)$ for different detuning values 374,368 and 360 .

(b) As (a) but for The first harmonic output field component $\left|\mathrm{x}_{+}\right|$. 


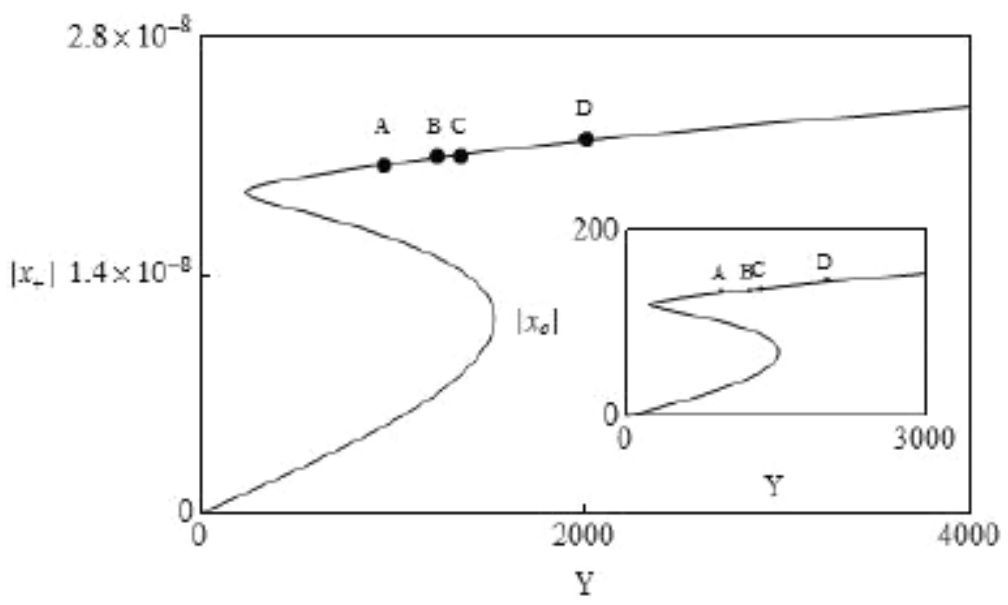

Fig. 1. The first harmonic output field component $\left|x_{+}\right|$against the incident field $Y$ for $C=70000$, $\kappa=0.25, \lambda=10^{-6}$ for $\delta=374, \theta=340$. Inset shows the fundamental output field component $\left|x_{0}\right|$ against the incident field $Y$. 


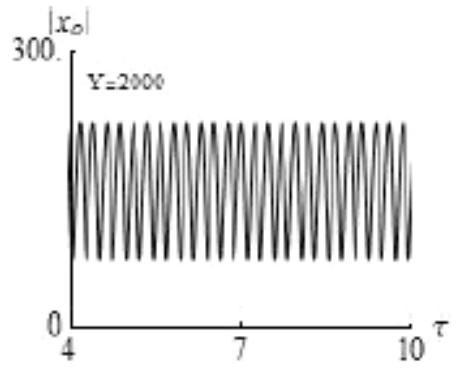

(a)

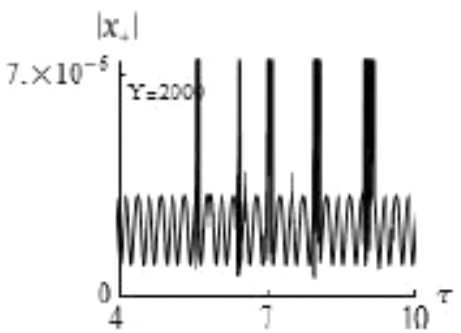

(c)

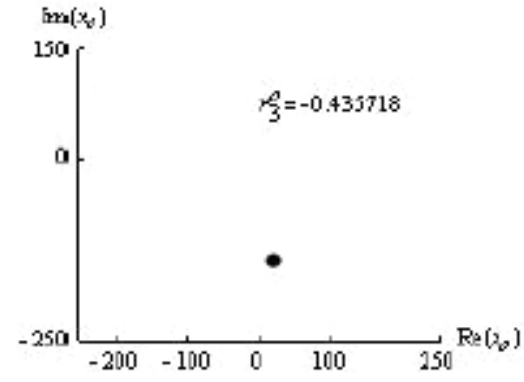

(e)

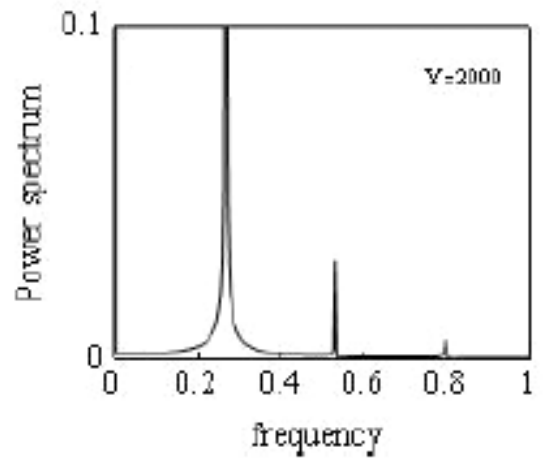

(g)

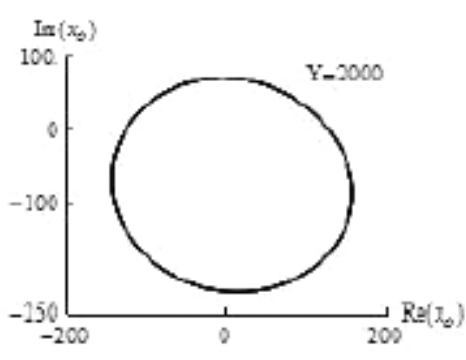

(b)

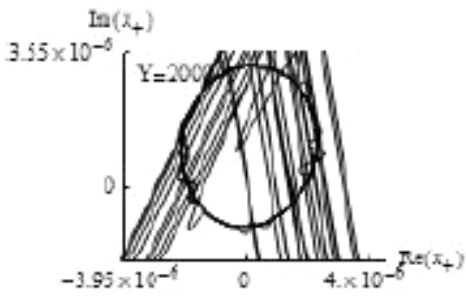

(d)

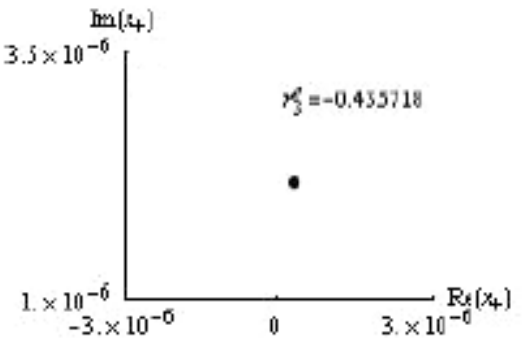

(f)

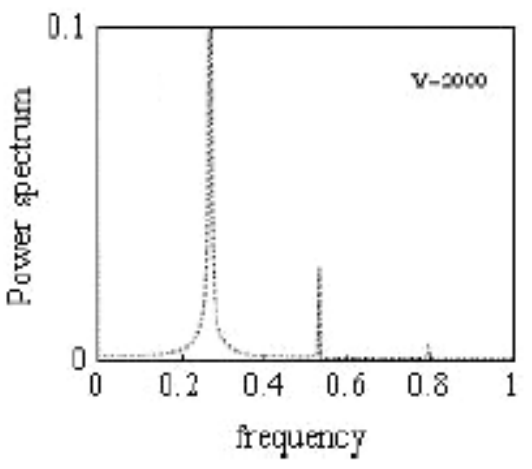

(h)

Fig. 2. (a, b) Self-pulse oscillations for both $\left|x_{\circ}\right|$ and $\left|x_{+}\right|$components in the adiabatic case against the time $\tau=\kappa t$ for $C=70000, \kappa=0.25, \lambda=10^{-6}, \delta=374, \theta=340$ and $Y=2000$. (c, d) The corresponding phase-space representation $\left(\operatorname{Re}\left(x_{\circ}\right), \operatorname{Im}\left(x_{\circ}\right)\right)$ for the fundamental output field component and $\left(\operatorname{Re}\left(x_{+}\right), \operatorname{Im}\left(x_{+}\right)\right)$for the first harmonic output field component, respectively. (e, f) Poincaré map for both $\left|x_{\mathrm{o}}\right|$ and $\left|x_{+}\right|$. (g, h) Power spectrum for both $\left|x_{\mathrm{o}}\right|$ and $\left|x_{+}\right|$. 


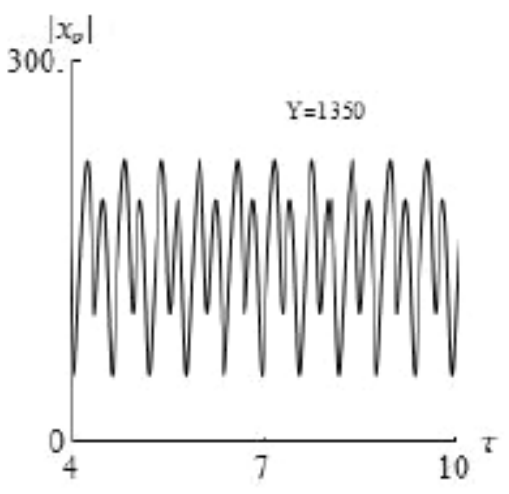

(a)

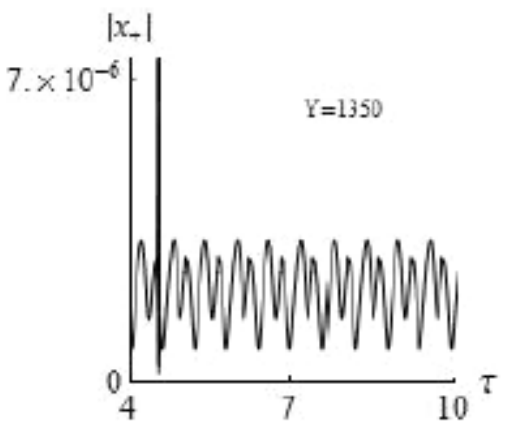

(c)

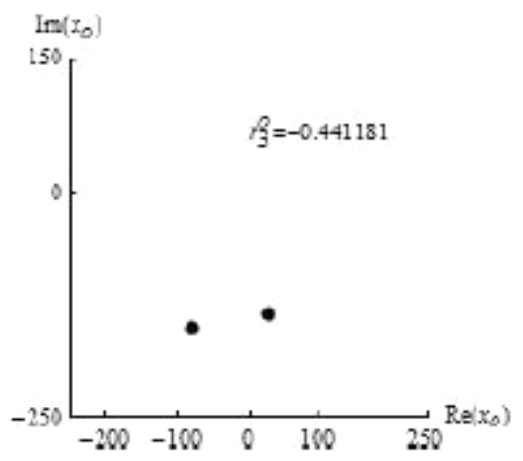

(e)

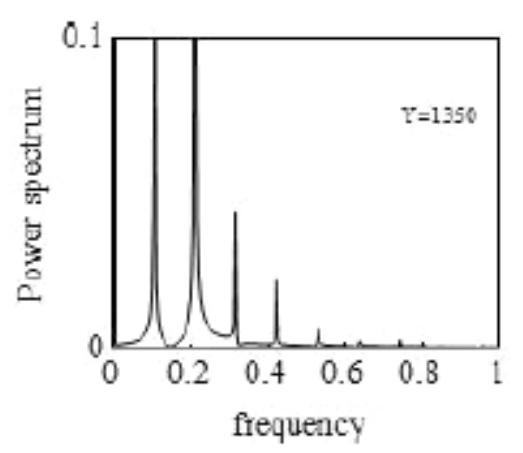

(g)

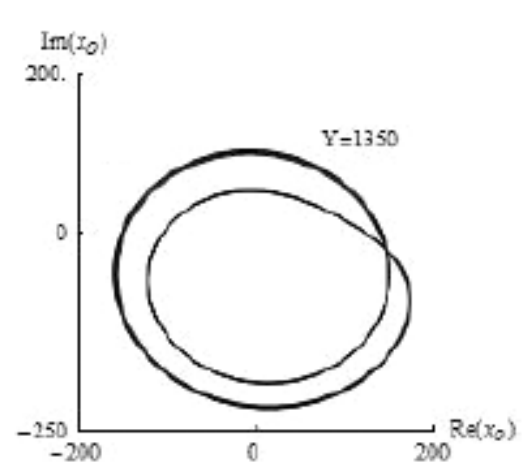

(b)

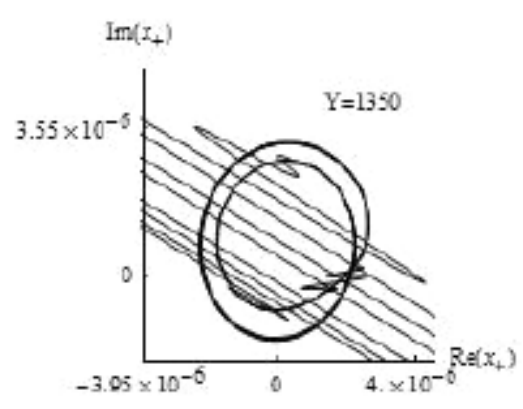

(d)

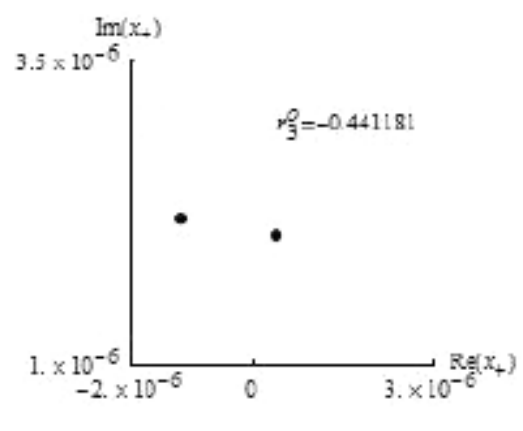

(f)

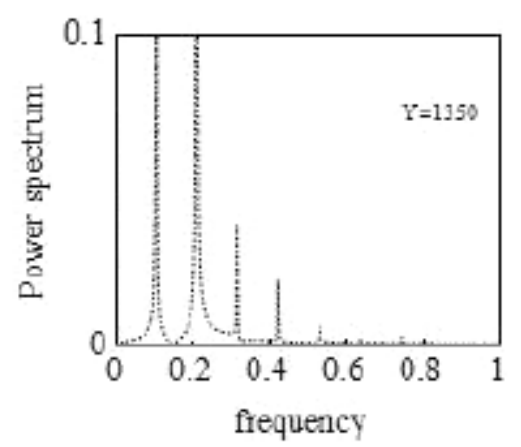

(h)

Fig. 3. Same as (2) but with $Y=1350$. 


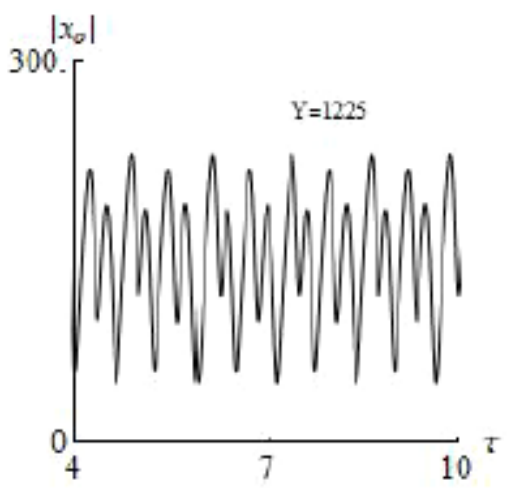

(a)

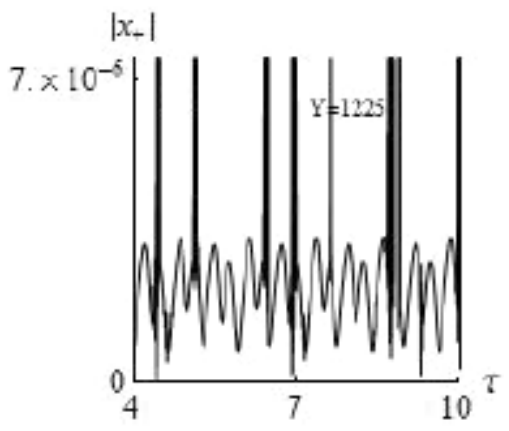

(c)

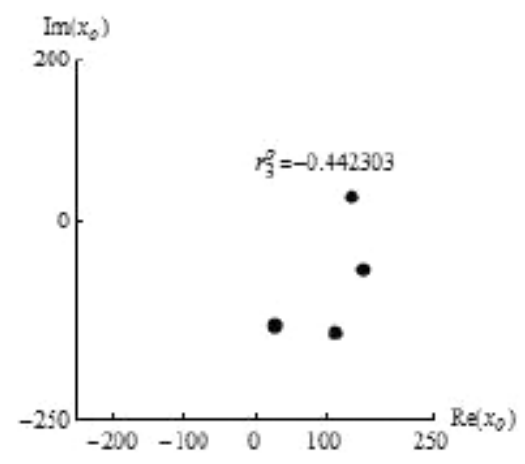

(e)

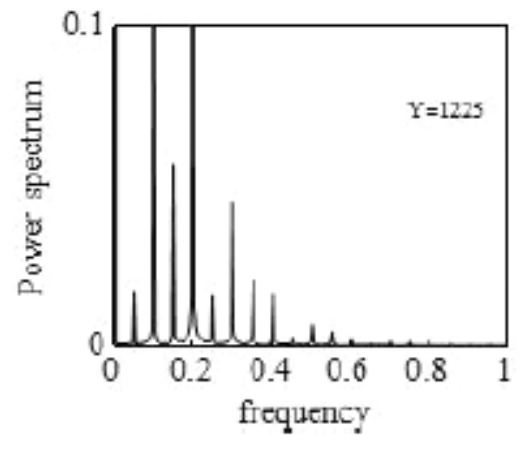

(g)

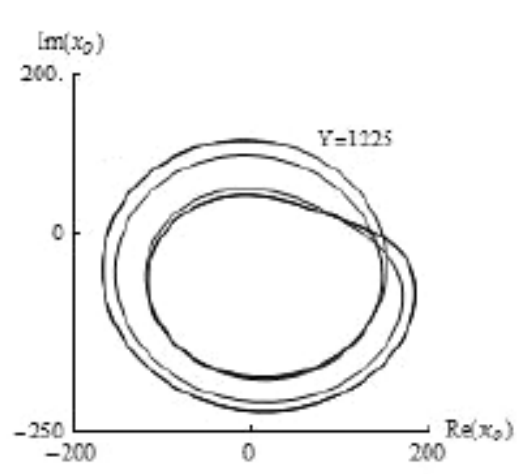

(b)

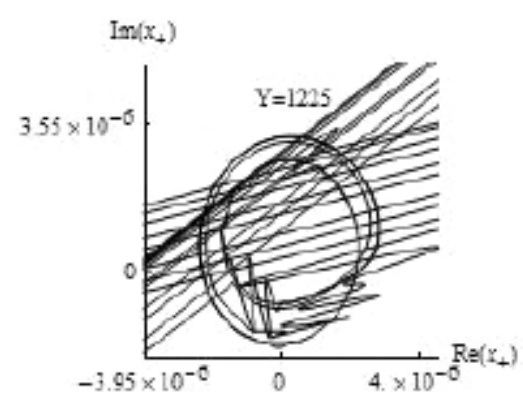

(d)

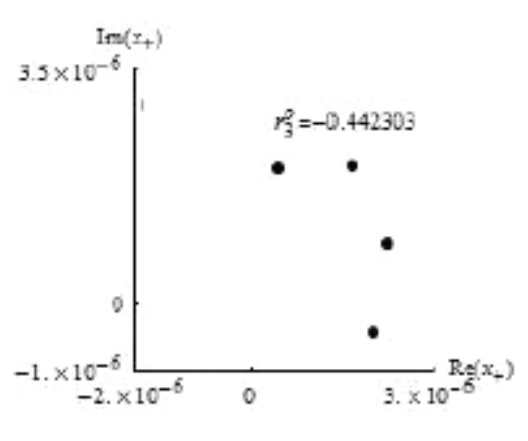

(f)

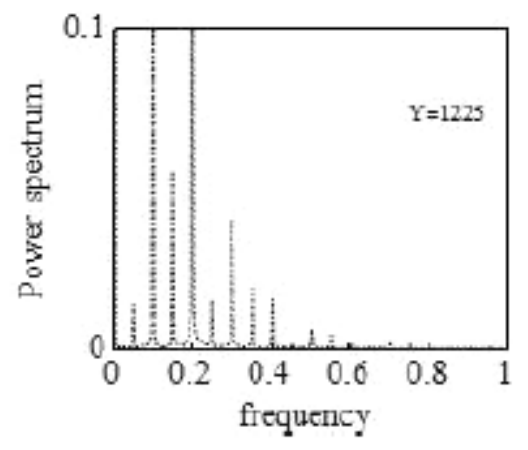

(h)

Fig. 4. Same as (2) but with $Y=1225$. 


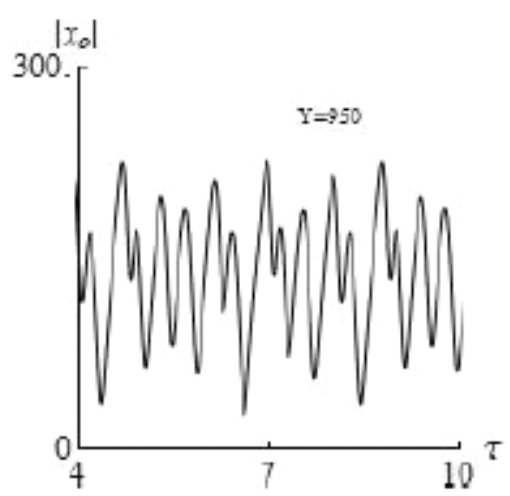

(a)

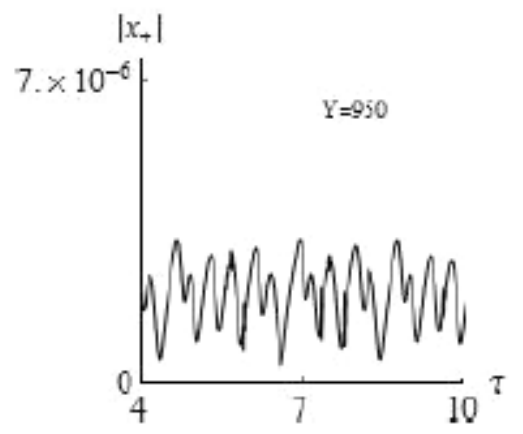

(c)

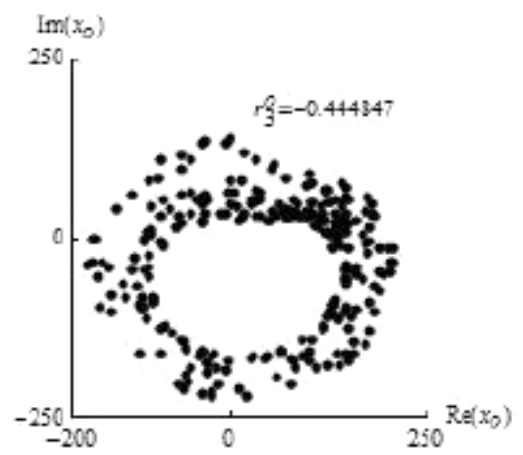

(e)

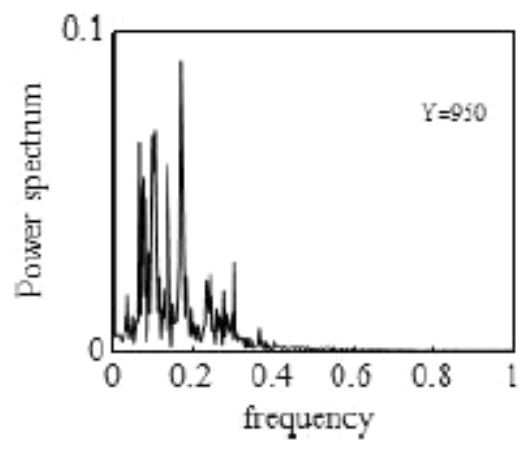

(g)

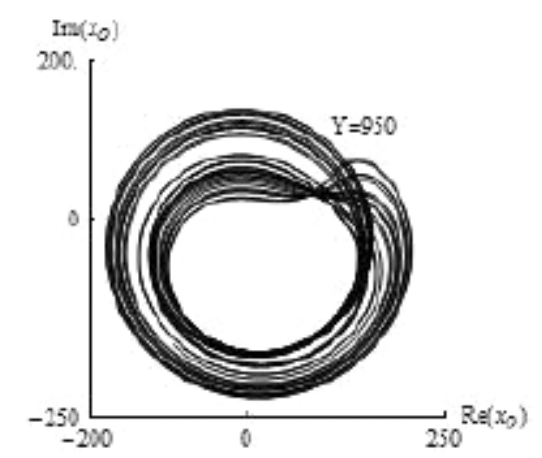

(b)

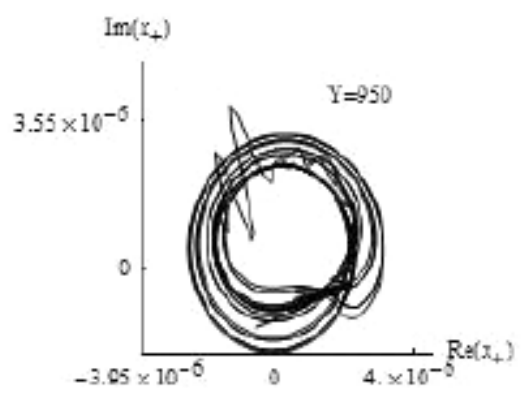

(d)

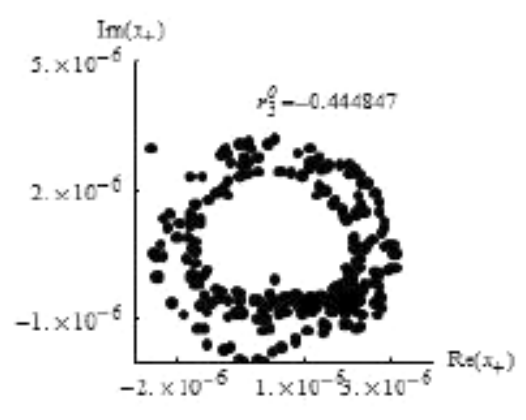

(f)

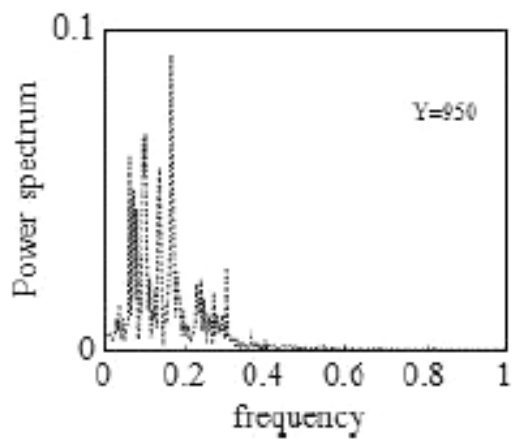

(h)

Fig. 5. Same as (2) but with $Y=950$. 


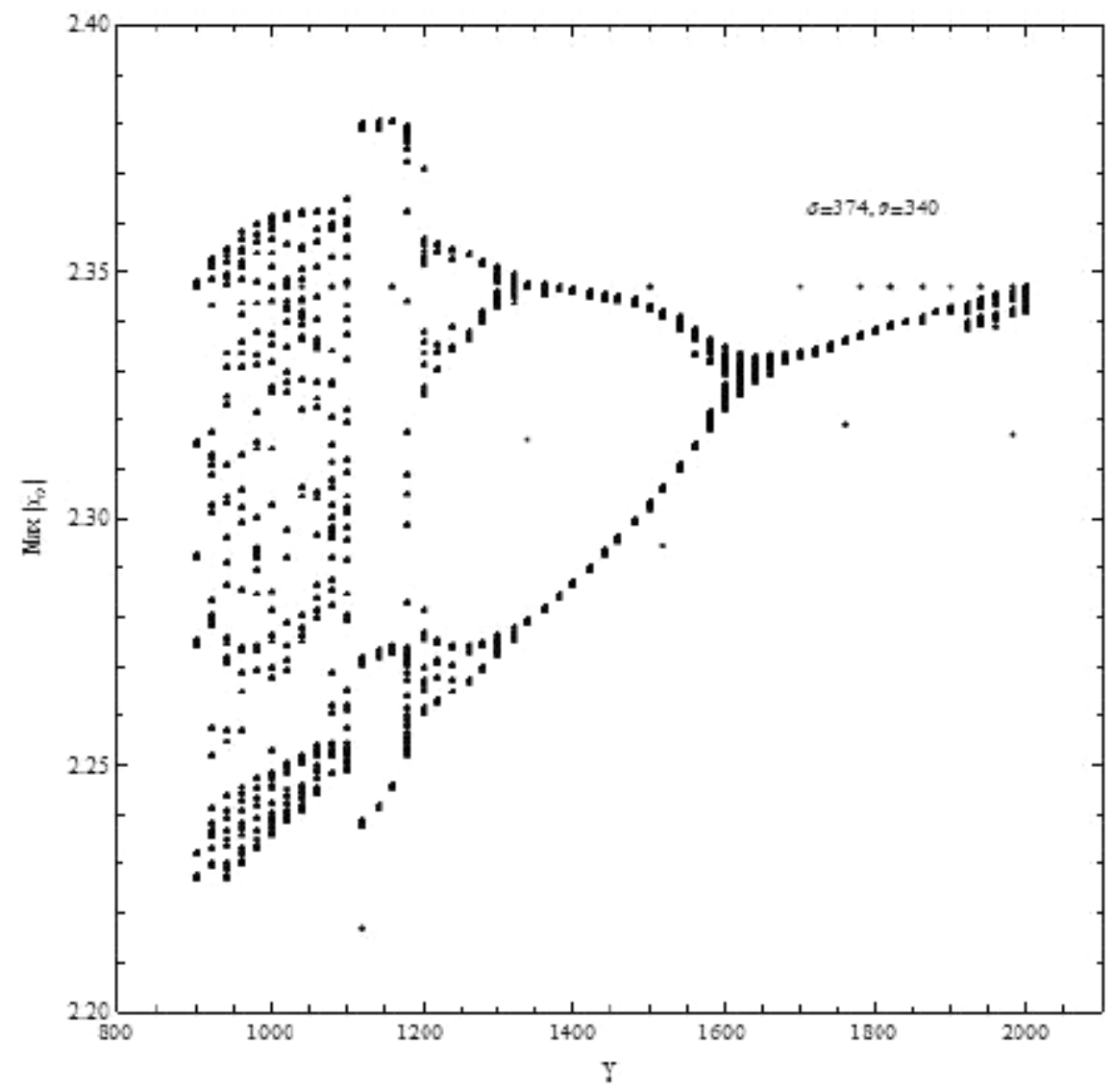

(a)

Fig. 6. (a) Bifurcation diagram representing the maximum value of the output field $\left|x_{\circ}\right|$ within RWA against the input field $Y$ for fixed $C=70,000, \delta=374$ and $\theta=340$. (b) As (a) but for the first harmonic output field component $\left|x_{+}\right|$for $\kappa=0.25$ and $\lambda=10^{-6}$. 


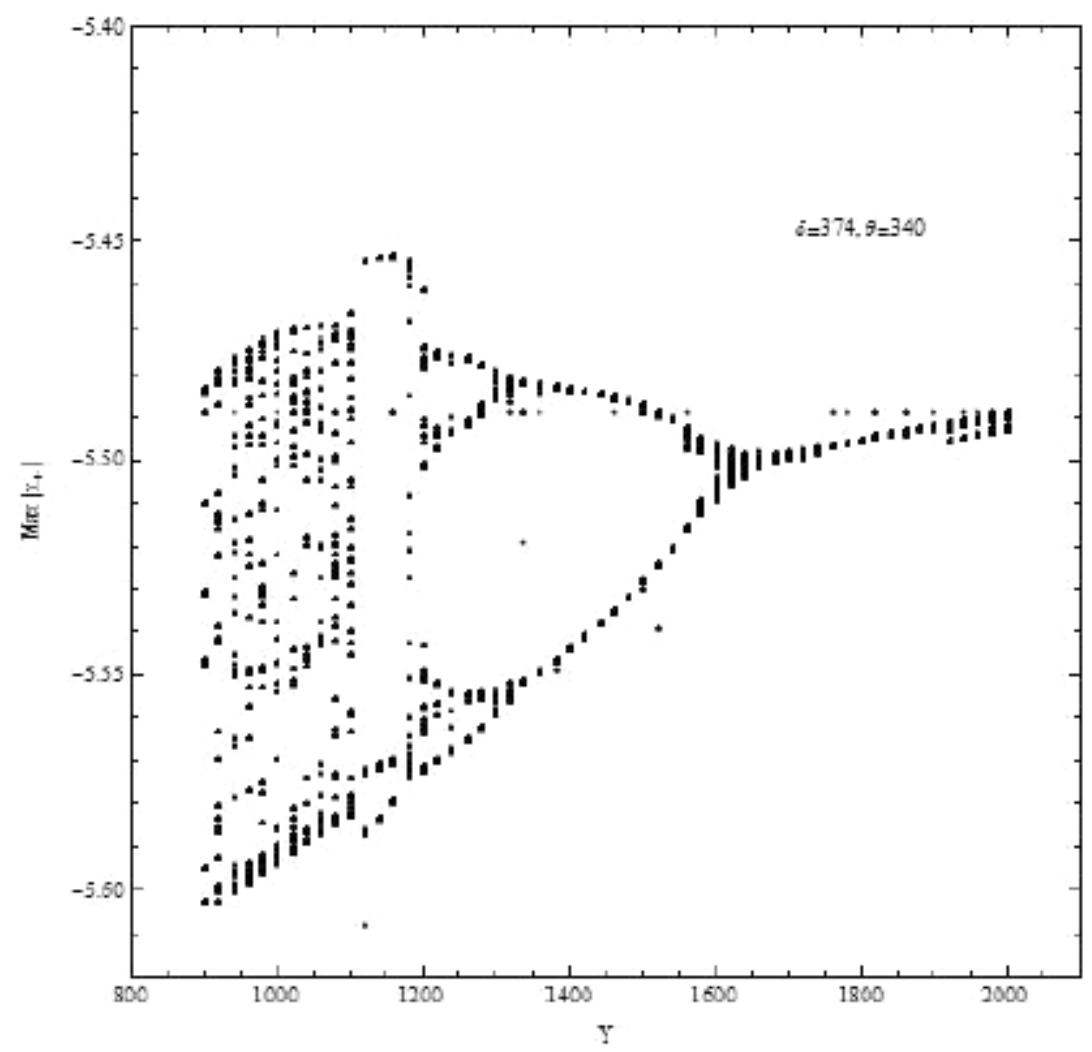

(b)

Fig. 6. (Continued)

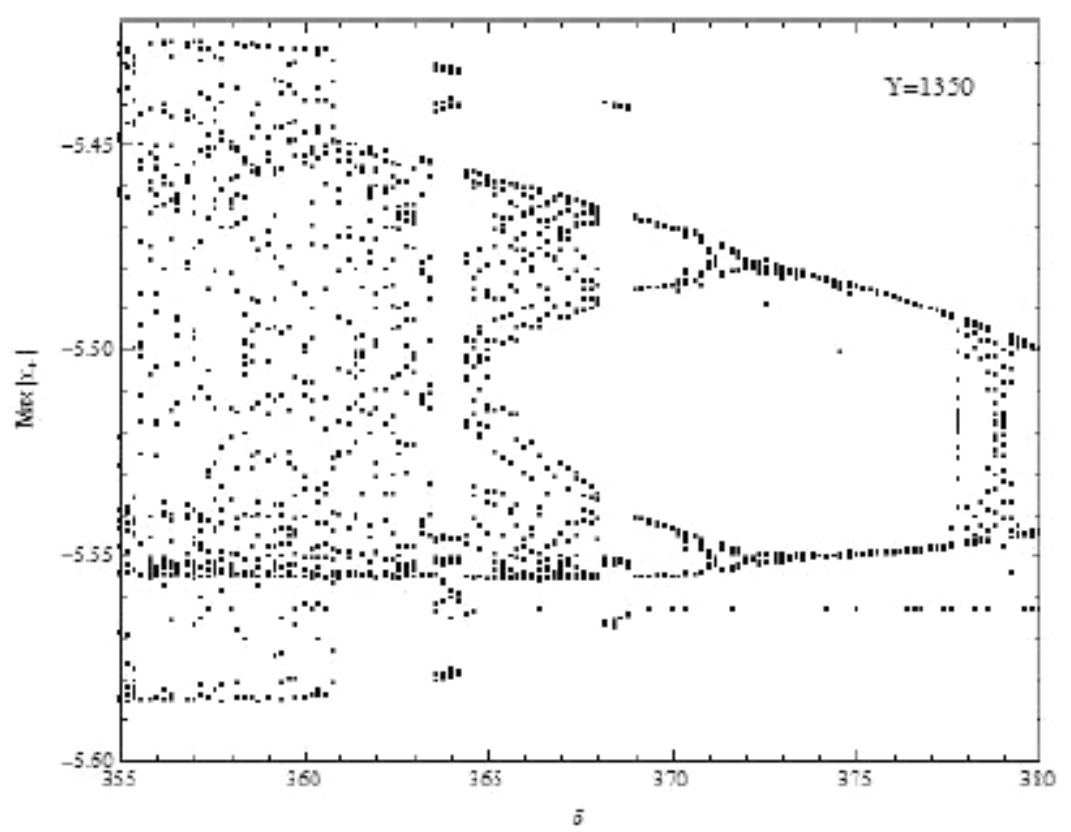

Fig. 7. Bifurcation diagram that represent the maximum value of the first harmonic output field component $\left|x_{+}\right|$against the atomic detuning $\delta$ for fixed values of other parameters as in Fig. 1, but with $Y=1350$. 


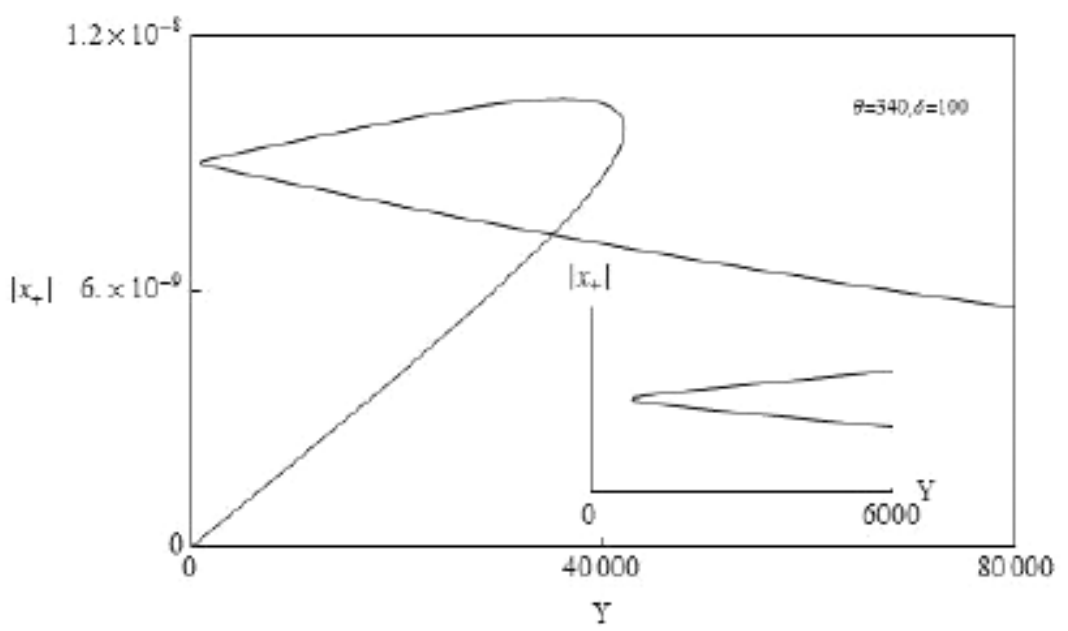

Fig. 8. Same as Fig. 1, but with $\kappa=0.3$ and $\delta=100$.

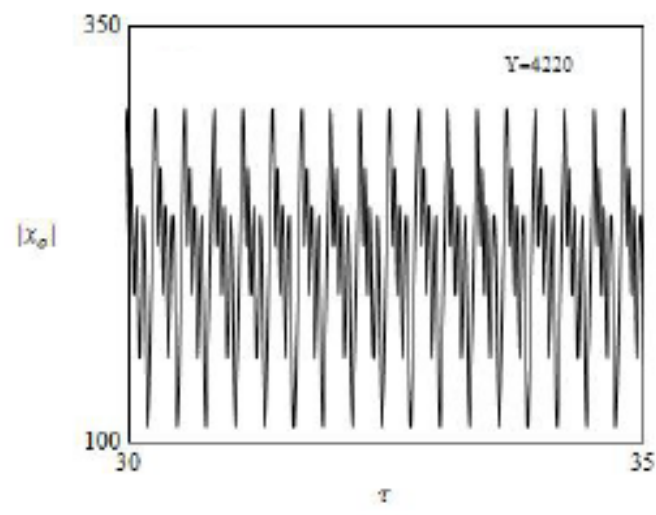

(a)

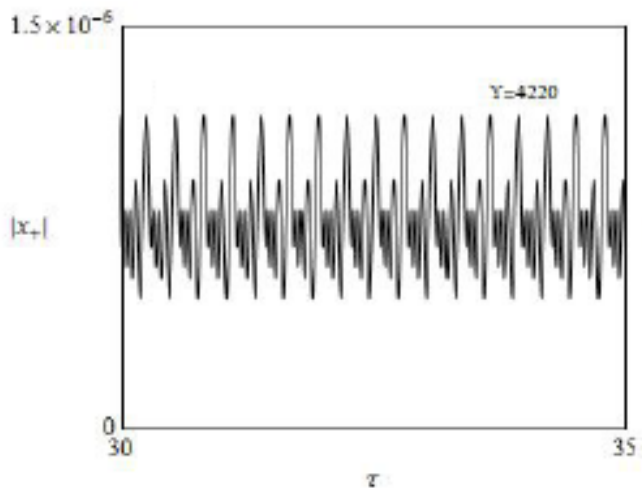

(c)

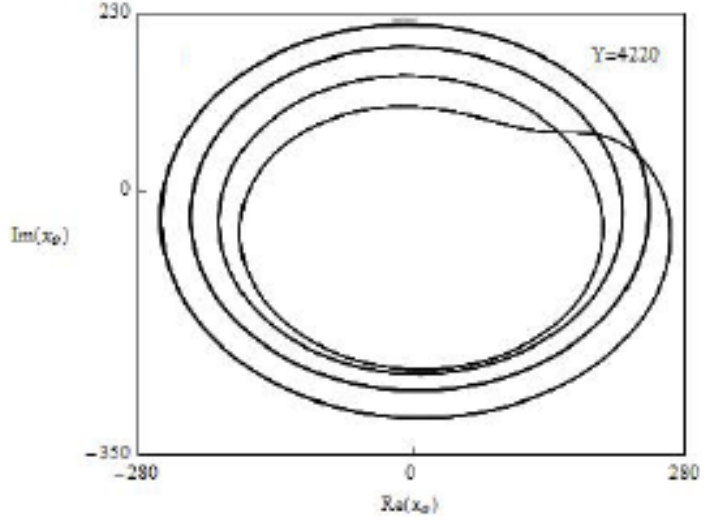

(b)

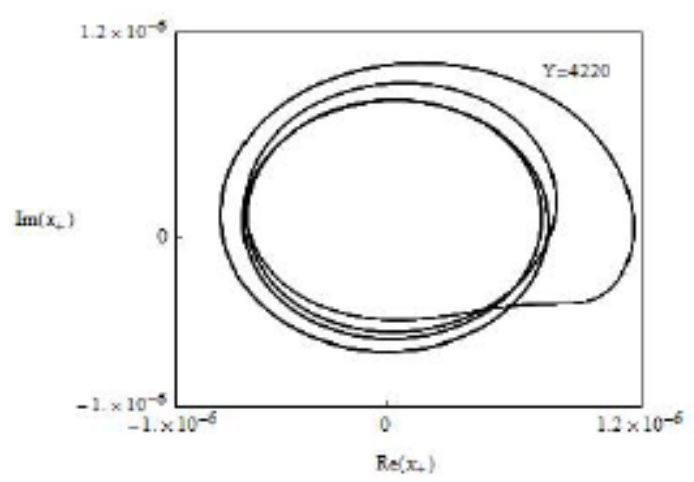

(d)

Fig. 9. (a-d) Same as (2.4a)-(2.4d), but with $\kappa=0.3, \delta=100, \theta=340$ and $Y=4220$. 
